part in the more elementary stages of physics similar to that which his well-known text-book has done and continues to do in the later stages.

Considering the amount of matter involved, it is, perhaps, not surprising that the treatment seems somewhat disjointed in the abrupt passages from one section to the next. This is most marked in the part on mechanics, which, however, the author tells us, is only included on account of the requirements in the main part of the book. As a consequence, it is doubtful whether it would be possible for a student to master the subject by unaided reading; but, after all, that should not be the purpose of a text-book. It should be regarded as an aid to oral instruction. The printing and diagrams are good and in many respects the book is unique.

(6) The fifth edition of Prof. Riecke's excellent text-book has been improved and extended in various ways. On one hand we have more complete treatment of such subjects as radio-activity and the conduction of electricity in gases, which in the earlier editions were only touched upon. On the other hand, there is the introduction of new matter, comprising liquid crystals, Brownian movements and the work of Michelson and of Nernst. Like most of the German standard textbooks, this one is much more complete than corresponding works in English, and it is greatly to the credit of the authors that they spare no trouble to bring the new editions of their books really up-to-date. The printing and diagrams are very much above the average. Surely there could be no better testimony of the worth of a scientific book than that it is now in its fifth edition.

(7) This book is quite a novelty. It consists of nearly fourteen hundred diagrams-curves, photographs, \&c.-representing physical facts, with short explanations of each collected at the end of the book. Although, of course, it could not be regarded as a substitute for an ordinary text-book, it might well be a useful companion to the latter. The arrangements of the diagrams is occasionally somewhat inelegant, doubtless due to space considerations, but they are, as a general rule, well printed, and in every case what is represented is clearly indicated.

\section{OUR BOOKSHELF}

Modern Problems. By Sir Oliver Lodge, F.R.S. Pp. vii +320 . (London: Methuen and Co., Ltd., r9i2.) Price $5 s$. net.

A COLLECTION of essays which have for the most part appeared before as articles or addresses, but which were well worth gathering together and publishing in more permanent form. The subjects dealt with are chiefly social and philosophic-the NO. 2244, VOL. 90] function of money, universal arbitration and the irrationality of war, Poor Law reform, the position of woman, the drink question, the nature of time, the philosophy of Bergson-these will indicate the wide scope of the book. All the chapters are characterised by the admirably lucid yet thorough exposition which Sir Oliver Lodge's writings always present; and the conclusions, definite though cool and undogmatic, are full of that ripe wisdom which only a wide human outlook on life can give.

From the scientific point of view, one of the most interesting chapters is that on the smoke nuisance, in which the author deals with the problems of combustion, and advocates the use of gas fires and the suppression of crude combustion of coal in towns. As to river and sea mists, and fogs of non-avoidable kind, Sir Oliver suggests electrification of the atmosphere on a large scale, a plan which he has brought within measurable distance of application. This matter is again touched on in the chapter "Squandering a Surplus." No one can tell for certain what would happen by this atmospheric electrification, but it is possible and even probable that the results might be of incalculable benefit; crops might be assisted, rain produced, fog dissipated. When we think of the tremendous harmfulness of fog, financially and to the health of our citizens, it seems obvious that the prospect of a cure of this evil would justify a large national grant for expenditure on trials in a large way. It is to be hoped something of the sort may yet be done. J. A. H.

Chrysanthemums. By T. Stevenson. With Chapters by C. H. Payne and C. E. Shea. Pp. xiv + I 12 . (London and Edinburgh: T. C. and E. C. Jack, n.d.) Price Is. $6 d$. net.

THIS is the latest addition to the admirable "Present-Day Gardening" series, edited by Mr. R. Hooper Pearson. It appears at an appropriate time and will interest as well as instruct all who are concerned with the culture of chrysanthemums, whether for pleasure or profit. Though there is no reference to the existence of the plant in English gardens before 1764 , it was mentioned by Confucius five hundred years or so before the commencement of our era. In the first third of the nineteenth century, about fifty varieties were known to English growers; and no attempt had been made to raise new varieties from seed. The first novelties obtained from seeds were exhibited by $\mathrm{Mr}$. I. Wheeler, of $\mathrm{Oxford}$, in $\mathrm{I}_{32}$; and since then hundreds of beautiful forms have been produced. In the volume before us, details are given as to the procedure in raising seedlings and creating new varieties; and also particulars as to the care of chrysanthemums in all stages of their growth. The book will delight and assist all growers of the plant, and is a valuable addition to a series which should be known to all lovers of gardening. There are eight coloured plates showing typical flowers, and lists of varieties for cultivation as decorative plants or flowers. 